\title{
Melatonin Suppresses Iron-Induced Apoptosis by Activating the Nrf2/HO-1 Signaling Pathway in Osteoblast
}

Hongdong Ma

Affiliated Hospital of Nantong University

\section{Zhiqing Li}

Qingdao Center Hospital: Qingdao Center Medical Group

Jiao Chu

Qingdao Center Hospital: Qingdao Center Medical Group

Dali Yin (D 406586503@qq.com)

Qingdao Center Hospital: Qingdao Center Medical Group https://orcid.org/0000-0002-5826-0345

\section{Research Article}

Keywords: Melatonin, Iron, Osteoblasts, Nrf2 signaling pathway, Cell apoptosis

Posted Date: February 19th, 2021

DOl: https://doi.org/10.21203/rs.3.rs-211800/v1

License: (c) (i) This work is licensed under a Creative Commons Attribution 4.0 International License. Read Full License 


\section{Abstract}

Objective To investigate the effect of melatonin on the apoptosis of hFOB1.19 cells induced by excess iron.

Methods The hFOB1.19 cells were treated with ferric ammonium citrate $(300 \mu \mathrm{mol} / \mathrm{L})$ and melatonin $(100$ $\mu \mathrm{mol} / \mathrm{L}$ ) for $24 \mathrm{~h}$. The apoptosis rate and the level of reactive oxygen species (ROS) were analyzed using flow cytometry. Expression of proteins associated with apoptosis, such as Bax and caspase-3, and those associated with the Nrf-2 signaling pathway such as Nrf2 and HO-1 were analyzed using western blotting. Results The level of ROS and the apoptosis rate increased after intervention with excess iron. The levels of Bax in the mitochondria and cleaved caspase-3 in the cytosol increased. However, after pretreatment with melatonin, the level of ROS, apoptosis rate, and expression of apoptosis-associated proteins decreased, and the expression of $\mathrm{Nrf} 2$ and $\mathrm{HO}-1$ increased.

Conclusion Melatonin inhibits the level of oxidation in osteoblasts via the Nrf2/HO-1 signal pathway, resulting in the reduction of apoptosis induced by excess iron.

\section{Introduction}

In recent years, the incidence of osteoporosis has increased, posing a serious threat to human health. Osteoporosis can easily lead to a series of adverse events, and cause serious consequences including disability and even death, bringing significant economic and social burdens to human society. A large number of studies have confirmed that osteoporosis and iron overload are obviously related ${ }^{1}$. Some researchers have proved that iron overload in the body has a toxic effect on osteoblasts ${ }^{2,3}$. By inhibiting the proliferation and differentiation of osteoblasts and thus exacerbating bone resorption, other studies have shown that patients with iron overload diseases have a higher incidence of osteoporosis ${ }^{4}$. However, in patients with osteoporosis, the process of bone microstructural changes and the exact mechanism of osteoblast iron overload are still unclear.

As an antioxidant, melatonin (MT) has a powerful and comprehensive free radical scavenging effect. It plays a beneficial role in sleep and circadian rhythm abnormalities, mood disorders, learning and memory, neuroprotection, drug abuse, and cancer ${ }^{5-7}$. In addition to the pineal gland secreting MT, studies have shown that MT can be synthesized in bone marrow and many other tissues ${ }^{8}$. The concentration of MT synthesized in bone marrow is twice that of night blood levels, suggesting that it may be related to bone metabolism. Many recent studies have confirmed that MT promotes the proliferation and differentiation of osteoblasts, inhibits the formation of osteoclasts to maintain the steady state of bone metabolism, and plays a role in preventing and treating osteoporosis ${ }^{9}$. However, the effect and mechanism of MT on iron overload-related osteoporosis remain unclear.

The nuclear factor erythroid 2-related factor 2 (Nrf2) signaling pathway is directly downstream of reactive oxygen species (ROS) and regulates the transcription of antioxidant responsive element (ARE)-dependent 
genes to balance oxidative mediators and maintain cellular redox homeostasis. Recent research revealed that MT reduced kidney damage caused by diabetes and exerted neuroprotective effects by activating the Nrf2/heme oxygenase-1 (HO-1) pathway and increasing levels of the antioxidant enzymes HO-1 and $\mathrm{NAD}(\mathrm{P}) \mathrm{H}$ dehydrogenase ${ }^{10} 1$ (NQO1) ${ }^{11}$. Nrf2 has been reported to protect against osteoporosis by inhibiting apoptosis ${ }^{12}$. However, further studies are required to identify the potential mechanisms of the Nrf2/HO-1 pathway and apoptosis in the improvement of bone microstructure by MT. In this study, the protective effect of MT on osteoblasts was explored by studying the apoptotic effect of iron overload and the effect of MT on this activity.

\section{Materials And Methods}

\section{Cell culture}

The human fetal osteoblastic cell line hFOB 1.19 , kindly provided by Dr. M. Subramaniam ${ }^{13}$, was maintained in a 1:1 mixture of Ham's F12 Medium and Dulbecco's Modified Eagle Medium without phenol red (Gibco, Carlsbad, CA, USA), supplemented with 10\% fetal bovine serum (FBS) (HyClone, Logan, UT, USA) and $0.3 \mathrm{~g} / \mathrm{L} \mathrm{G} 418$ (Sigma-Aldrich, St. Louis, MO, USA), in a humidified $5 \% \mathrm{CO}_{2}$ atmosphere at $33.5^{\circ} \mathrm{C}$, with medium changes every other day. The cells were subcultured using trypsin-EDTA and replated for use in experiments. The hFOB 1.19 cells were plated at $10^{4}$ cells $/ \mathrm{cm}^{2}$ and allowed to adhere for $24 \mathrm{~h}$ before treatment.

\section{Reagents and antibodies}

The reagents 3-(4,5-dimethyl-thiazol-2-yl)-2,5-diphenyltetrazolium bromide (MTT), ferric ammonium citrate (FAC) and MT were obtained from Sigma-Aldrich. An annexin V-FITC/PI apoptosis detection kit was purchased from Beijing Seboc, while primary antibodies for Nrf2, HO-1, BAX, and cleaved caspase 3 were purchased from Abcam (Cambridge, MA, USA).

\section{Cell viability analysis}

Cell viability was measured using MTT. Briefly, the cells were seeded into 96 -well plates (6,000 cells/well), incubated for $24 \mathrm{~h}$, then the medium was replaced with medium containing $10 \%$ serum. After treatment, culture medium was changed for serum-free culture medium. MTT dissolved in phosphate-buffered saline (PBS) was added to each well and then incubated for $4 \mathrm{~h}$. After this interval, the serum-free culture medium containing MTT was discarded and dimethyl sulfoxide (DMSO) was added to each well to dissolve the precipitate. The optical densities were measured at a spectral wavelength of $490 \mathrm{~nm}$ using a microplate reader (Spectra Thermo, Switzerland). Cell proliferation was estimated using a BrdU kit (Roche, Basel, Switzerland) following the manufacturer's protocol. Cell viability and proliferation results were expressed as percentages. The absorbance measured from untreated cells was taken to be $100 \%$.

\section{Cell apoptosis analysis}


Cell apoptosis was determined by detecting phosphatidylserine exposure on the cellular plasma membranes using the fluorescent dye Annexin V-APC apoptosis detection kit with 7AAD according to the manufacturer's protocol. In brief, the cells were harvested, washed twice in ice-cold PBS, resuspended in $500 \mu \mathrm{L}$ of binding buffer, incubated with $5 \mu \mathrm{L}$ of Annexin V-APC and $5 \mu \mathrm{L}$ of 7AAD solution for $15 \mathrm{~min}$ at room temperature in the dark and then immediately analyzed by bivariate flow cytometry using an FAC Scan-LSR equipped with Cell Quest software. Approximately $5 \times 10^{5}$ cells were analyzed in each of the samples. The experiment was repeated three times.

\section{Determination of ROS levels}

Cells were seeded at $2 \times 10^{5}$ cells/well into 6 -well plates, and fed with $2 \mathrm{~mL}$ of medium supplemented with the different drugs. After incubation for $48 \mathrm{~h}$, the culture medium was replaced with serum-free medium containing $10 \mu \mathrm{mol} / \mathrm{L}$ 2,7-dichlorodihydrofluorescein diacetate (Sigma-Aldrich) and placed in the dark for $30 \mathrm{~min}$, with gentle shaking every $5 \mathrm{~min}$. The cells were then harvested in $15 \mathrm{~mL}$ tubes, centrifuged at 1,000 $\times g$ for 5 min and washed three times with serum-free medium, followed by resuspension in serum-free medium and incubation with $5 \mu \mathrm{L}$ 7-aminoactinomycin D (Key GEN Bio-TECH, Nanjing, China) in the dark for 5 min. Fluorescence was measured by flow cytometry (BD Biosciences, San Jose, CA, USA) at an excitation wavelength of $488 \mathrm{~nm}$ and an emission wavelength of $525 \mathrm{~nm}$. The average intensity of fluorescence in each group indicated the amount of ROS within the cells.

\section{Western blot analysis}

After treatment, the cells were extracted with lysis buffer (150 mm NaCl, $1 \% \mathrm{NP}-40,0.1 \%$ sodium dodecyl sulfate (SDS), $2 \mu \mathrm{g} / \mathrm{mL}$ aprotinin, $1 \mathrm{~mm}$ phenylmethylsulfonyl fluoride) for $30 \mathrm{~min}$ at $4^{\circ} \mathrm{C}$. The supernatant was centrifuged at $12,000 \times g$ for $15 \mathrm{~min}$ at $4^{\circ} \mathrm{C}$ and the supernatant containing total protein was harvested. Aliquots containing $50 \mu \mathrm{g}$ of protein were separated by $12 \%$ SDS-polyacrylamide gel electrophoresis and transferred to polyvinylidene difluoride membranes at 60 or $40 \mathrm{~V}$ for $2 \mathrm{~h}$ at low temperature. The membranes were soaked in blocking buffer ( $5 \%$ skimmed milk) for $2 \mathrm{~h}$ and proteins were then detected using primary antibodies at $1: 500$ or $1: 1,000$ dilution overnight at $4^{\circ} \mathrm{C}$, followed by visualization using anti-goat or anti-rabbit horseradish peroxidase-conjugated IgG secondary antibodies at 1:6,000 or 1:8,000 dilution for $2 \mathrm{~h}$ at room temperature. Specific bands were visualized using the EC3 Imaging System (UVP Inc. Upland, CA, USA), and the optical density of each band was measured using ImageJ software (NIH, Bethesda, MD, USA). The relative content was calculated as the ratio between the protein of interest and $\beta$-actin in the same sample, and expressed graphically.

\section{Statistical analysis}

Quantitative variables are expressed as the mean \pm standard deviation. All data were analyzed using GraphPad Prism 6.02 (GraphPad Software Inc., La Jolla, CA, USA). Differences between two groups were analyzed by Student's t-test and between multiple groups were compared by one-way analysis of variance (ANOVA). A $P$ value $<0.05$ was considered statistically significant.

\section{Results}




\section{Apoptosis was induced by FAC in osteoblastic hFOB1.19 cells.}

To determine the influence of FAC on the viability of osteoblasts, we treated human hFOB1.19 osteoblasts with different concentrations of $\operatorname{FAC}(0,50,100,200,300$, or $500 \mu \mathrm{mol} / \mathrm{L})$ for different time periods (12 or $24 \mathrm{~h}$ ), and then assessed the viability of the treated cells with a 3-(4,5- dimethylthiazol- 2yl)-2,5-diphenyltetrazolium bromide (MTT) assay (Fig. 1A). The viability of the cells treated with 300 $\mu \mathrm{mol} / \mathrm{L}$ FAC for $24 \mathrm{~h}$ decreased by $50 \%$ relative to that of the control cells. Consequently, an FAC concentration of $300 \mu \mathrm{mol} / \mathrm{L}$ was used for subsequent experiments $(P<0.001 \mathrm{vs}$. control cells).

To determine whether the inhibitory effect of $24 \mathrm{~h}$ treatment with $300 \mu \mathrm{mol} / \mathrm{L}$ FAC on osteoblast viability was related to apoptosis, an annexin V-APC/7AAD apoptosis detection kit was used. The DNA fragmentation ratio in the FAC-treated hFOB1.19 cells was higher than that the control group (Fig. 1B). We used western blotting analysis to measure levels of apoptosis-related proteins, and found that the expressions of the proapoptotic proteins BAX and cleaved caspase 3 were increased compared with the control group (Fig. 2A). These results indicated that FAC-induced apoptosis was the cause of the reduced cell viability in osteoblastic hFOB1.19 cells.

\section{MT protected hFOB1.19 cells against apoptosis via the Nrf2/HO-1 signaling pathway.}

We next investigated whether the reduction in hFOB1.19 cell viability caused by FAC-induced apoptosis could be rescued by MT supplementation. The FAC-induced decrease in cell viability was reversed by 100 $\mu \mathrm{M}$ MT, as determined by MTT assay (Fig. 1A). Furthermore, we found that MT reduced the FAC-induced inhibition of cell viability and inhibited apoptotic cell death (Fig. 1B). Treatment of cells with MT significantly reduced the protein concentrations of caspase 3 and BAX, indicating that MT inhibited apoptosis in hFOB1.19 cells (Fig. 2A).

As an antioxidant, MT has been widely used to treat various diseases. Flow cytometry was used to detect ROS levels after FAC and MT intervention. The results showed that ROS levels increased significantly after FAC intervention, but this phenomenon was significantly reversed by MT (Fig. 2B). In order to prove the relationship between the Nrf2/ HO-1 signaling pathway and MT inhibition of apoptosis, we analyzed the expression levels of Nrf2 and HO-1 in osteoblasts by western blotting. The results showed that, compared with the FAC group, the expression levels of Nrf2 and HO-1 were significantly increased after addition of MT (Fig. 2C), which suggested that MT protected hFOB1.19 cells against apoptosis via the $\mathrm{Nrf} 2 / \mathrm{HO}-1$ signaling pathway.

\section{Discussion}

Osteoporosis is the metabolic bone disease with the highest incidence, which seriously threatens patients' safety and quality of life. With the aging of the population, the incidence and mortality of osteoporosis, as well as the resulting economic burden, have increased significantly. Osteoporosis is a chronic bone disease characterized by bone tissue degradation, bone loss, and easy fracture. The imbalance between osteoblasts and osteoclasts is the direct cause of this pathological process. 
Abnormal differentiation of both these cell types plays an important role in the pathogenesis of osteoporosis. In patients with osteoporosis, osteoblast differentiation decreases and osteoclast differentiation increases, which ultimately leads to abnormal bone mass.

Iron is an essential trace element for the human body and the ions have important biological functions. When the amount of iron ions in the blood exceeds the binding capacity of transferrin, it forms free iron that is not bound by transferrin, leading to "iron overload."

Osteoblasts are the main functional cells of bone formation and originate from pluripotent bone marrow stromal cells. These cells pass through four life stages during bone formation: osteoblast proliferation, extracellular matrix maturation, extracellular matrix mineralization and osteoblast apoptosis ${ }^{14}$.

Some studies have shown that iron overload can lead to osteoporosis in rats due to oxidation ${ }^{15}$. Recent studies have shown that iron overload inhibits osteoblast function by increasing intracellular oxidative stress, and at the same time promotes osteoclast differentiation and bone resorption by generating reactive oxygen species ${ }^{3,4,16}$. This suggests that the effect of iron overload on osteoblasts may be related to oxidative stress. In this study, we found that $300 \mu \mathrm{mol} / \mathrm{L}$ FAC significantly increased the level of ROS in osteoblasts and caused osteoblast apoptosis, which indicated that high iron increased the level of intracellular ROS, causing cell oxidative stress damage and inducing osteoblast apoptosis.

MT is a neuroendocrine hormone, mainly secreted by the pineal gland, which has a variety of physiological effects including regulation of the biological rhythm, improving sleep quality, increasing immunity, and exerting anti-oxidative stress, anti-tumor, and anti-aging effects ${ }^{17,18}$. Recent studies have shown that MT is closely related to senile osteoporosis ${ }^{19}$. We found that $100 \mu \mathrm{mol} / \mathrm{L} \mathrm{MT} \mathrm{significantly}$ inhibited the apoptosis of osteoblasts induced by high iron, indicating that MT has a certain protective effect on osteoblasts. At the same time, $100 \mu \mathrm{mol} / \mathrm{L} \mathrm{MT}$ significantly reduced the increase in ROS levels observed in osteoblasts caused by $300 \mu \mathrm{mol} / \mathrm{L} \mathrm{FAC}$, and reduced the oxidative stress of osteoblasts. Some researchers proposed that MT is an antioxidant and discussed its antioxidant mechanism.

Subsequently, there have been other reports that MT clears other active oxygen components such as lipid peroxidation free radicals (LOO-) and hydrogen peroxide ${ }^{20,21}$. MT has shown a powerful and comprehensive free radical scavenging effect. In addition, it is used in a variety of oxidative damage animal models where protective effects of MT on superoxide dismutase (SOD) and glutathione oxidase (GSH-Px) in tissues have been observed ${ }^{22}$. These all confirmed the antioxidant effect of MT. Our further experiments revealed that $100 \mu \mathrm{mol} / \mathrm{L}$ MT significantly increased the expression of Nrf-2 and HO-1 in cells, indicating that MT can activate the antioxidant system in osteoblasts by activating Nrf-2 related pathways. Nrf-2 is a positive regulator of the human antioxidant response element, which promotes $\mathrm{NAD}(\mathrm{P}) \mathrm{H}$, quinone oxidoreductase, and heme monooxygenase. The expression of various antioxidant enzymes such as glutathione peroxidase and $\gamma$-glutamylcysteine synthetase. The Nrf2/HO-1 pathway is the cell's defense against exogenous stimulation and one of the main mechanisms of oxidative damage. The Nrf2/HO-1 signaling pathway and its downstream genes have been proven to play an important role in the anti-oxidative damage of a variety of cells, tissues and organs ${ }^{23}$. Activation of the Nrf-2 pathway 
can be regulated the expression level of a large number of antioxidant gene products in the cell, thus reducing the level of oxidative stress, and the pathophysiological changes related to oxidative stress. MT promotes the elimination of oxidative free radicals in osteoblasts by activating the Nrf2 pathway, thereby inhibiting the increase in intracellular ROS levels caused by iron overload, and reducing the oxidative stress damage of osteoblasts.

In summary, studies have shown that MT reduced the increase of ROS caused by high iron by activating the intracellular Nrf2/HO-1 pathway, and reduced the level of osteoblast apoptosis, thereby protecting against osteoporosis caused by oxidative stress.

\section{Declarations}

\section{Acknowledgements}

Not applicable.

\section{Funding}

No funding was received.

\section{Availability of data and materials}

The datasets used and/or analyzed during the current study are available from the corresponding author on reasonable request.

\section{Authors' contributions}

Dali Yin conceived and designed the study. Hongdong Ma performed data analyses and wrote the manuscript. Zhiqing Li and Jiao Chu contributed to data acquisition and manuscript revision.

\section{Ethics approval and consent to participate}

Not applicable.

\section{Patient consent for publication}

Not applicable.

\section{Competing interests}

The authors declare that they have no competing interests.

\section{References}


1. Jing X, Du T, Chen K et al (2019) Icariin protects against iron overload-induced bone loss via suppressing oxidative stress. Journal of cellular physiology 234:10123-10137

2. Zhang J, Qiao P, Yao G, Zhao H, Wu Y, Wu S (2020) lonizing Radiation Exacerbates the Bone Loss Induced by Iron Overload in Mice. Biol Trace Elem Res 196:502-511

3. Tian Q, Qin B, Gu Y, et al. ROS-Mediated Necroptosis Is Involved in Iron Overload-Induced Osteoblastic Cell Death. Oxidative medicine and cellular longevity. 2020; 2020: 1295382

4. Piriyakhuntorn P, Tantiworawit A, Phimphilai M, Shinlapawittayatorn K, Chattipakorn S, Chattipakorn $N$ (2020) Impact of iron overload on bone remodeling in thalassemia. Archives of osteoporosis $15: 143$

5. Karamitri A, Jockers R (2019) Melatonin in type 2 diabetes mellitus and obesity. Nature reviews Endocrinology 15:105-125

6. Cipolla-Neto J, Amaral F (2018) Melatonin as a Hormone: New Physiological and Clinical Insights. Endocr Rev 39:990-1028

7. Xia Y, Chen S, Zeng S et al (2019) Melatonin in macrophage biology: Current understanding and future perspectives. Journal of pineal research 66:e12547

8. Sharan K, Lewis K, Furukawa T, Yadav V. Regulation of bone mass through pineal-derived melatoninMT2 receptor pathway. Journal of pineal research. 2017; 63

9. Zhao R, Tao L, Qiu S et al (2020) Melatonin rescues glucocorticoid-induced inhibition of osteoblast differentiation in MC3T3-E1 cells via the PI3K/AKT and BMP/Smad signalling pathways. Life sciences 257:118044

10. Delagrange P, Boutin JA (2006) Therapeutic potential of melatonin ligands. Chronobiol Int 23:413418

11. Zhang D (2006) Mechanistic studies of the Nrf2-Keap1 signaling pathway. Drug metabolism reviews 38:769-789

12. Han D, Gu X, Gao J et al (2019) Chlorogenic acid promotes the Nrf2/HO-1 anti-oxidative pathway by activating p21 to resist dexamethasone-induced apoptosis in osteoblastic cells. Free Radic Biol Med $137: 1-12$

13. Subramaniam M, Jalal S, Rickard D, Harris S, Bolander M, Spelsberg $T$ (2002) Further characterization of human fetal osteoblastic hFOB 1.19 and hFOB/ER alpha cells: bone formation in vivo and karyotype analysis using multicolor fluorescent in situ hybridization. Journal of cellular biochemistry 87:9-15

14. Yang J, Dong D, Luo X, Zhou J, Shang P, Zhang H (2020) Iron Overload-Induced Osteocyte Apoptosis Stimulates Osteoclast Differentiation Through Increasing Osteocytic RANKL Production In Vitro. Calcif Tissue Int 107:499-509

15. Zhao L, Wang Y, Wang Z, Xu Z, Zhang Q, Yin M (2015) Effects of dietary resveratrol on excess-ironinduced bone loss via antioxidative character. J Nutr Biochem 26:1174-1182 
16. Fang S, Yu X, Ding H, Han J, Feng J (2018) Effects of intracellular iron overload on cell death and identification of potent cell death inhibitors. Biochem Biophys Res Commun 503:297-303

17. Xie Z, Chen F, Li W et al (2017) A review of sleep disorders and melatonin. Neurological research 39:559-565

18. Liu Z, Gan L, Luo D, Sun C. Melatonin promotes circadian rhythm-induced proliferation through Clock/histone deacetylase 3/c-Myc interaction in mouse adipose tissue. Journal of pineal research. $2017 ; 62$

19. Li T, Jiang S, Lu C et al (2019) Melatonin: Another avenue for treating osteoporosis? Journal of pineal research 66:e12548

20. Patiño P, Parada E, Farré-Alins V et al (2016) Melatonin protects against oxygen and glucose deprivation by decreasing extracellular glutamate and Nox-derived ROS in rat hippocampal slices. Neurotoxicology 57:61-68

21. Najafi A, Adutwum E, Yari A et al (2018) Melatonin affects membrane integrity, intracellular reactive oxygen species, caspase 3 activity and AKT phosphorylation in frozen thawed human sperm. Cell tissue research 372:149-159

22. Goc Z, Szaroma W, Kapusta E, Dziubek K (2017) Protective effects of melatonin on the activity of SOD, CAT, GSH-Px and GSH content in organs of mice after administration of SNP. Chin J Physiol 60:1-10

23. Chen X, Xi Z, Liang H et al (2019) in vitroMelatonin Prevents Mice Cortical Astrocytes From HeminInduced Toxicity Through Activating PKCa/Nrf2/HO-1 Signaling. Front NeuroSci 13:760

\section{Figures}


A
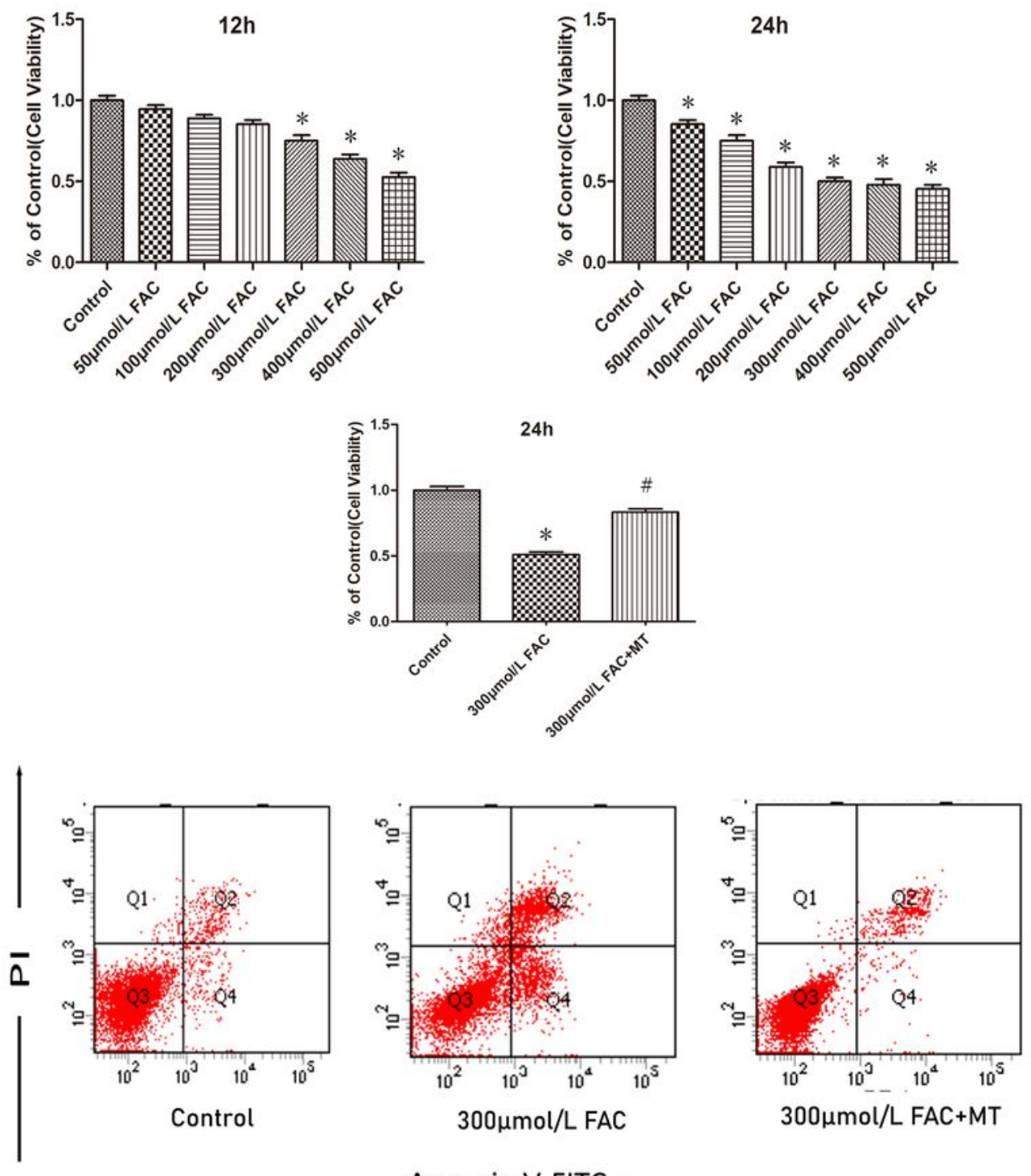

Annexin V-FITC

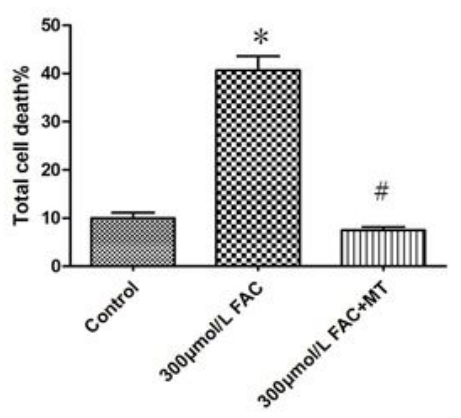

\section{Figure 1}

Apoptosis was induced by FAC in osteoblastic hFOB1.19 cells. A. Cells were treated with various concentrations of FAC for $12 \mathrm{~h}$ or $24 \mathrm{~h}$. Cells were then treated with or without $100 \mu \mathrm{mol} / \mathrm{L}$ melatonin and incubated with $300 \mu \mathrm{mol} / \mathrm{L}$ FAC for $24 \mathrm{~h}$. B. Cells were collected, stained with annexin V-APC/7AAD, and examined by flow cytometry. The histogram shows the apoptosis rate in each group. Values represent the 
mean \pm S.E.M. of at least three independent experiments. ${ }^{*} \mathrm{P}<0.05$ vs. control, \#P $<0.05$ vs. FAC treatment.

\section{A}

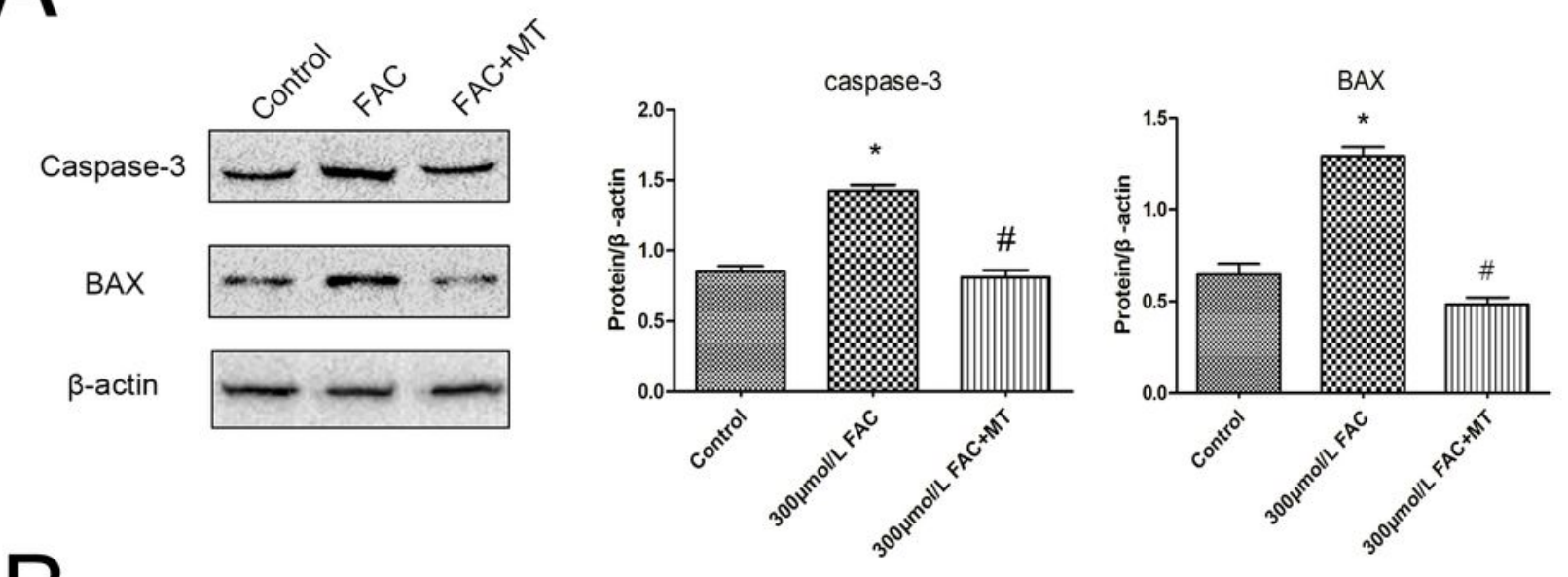

B
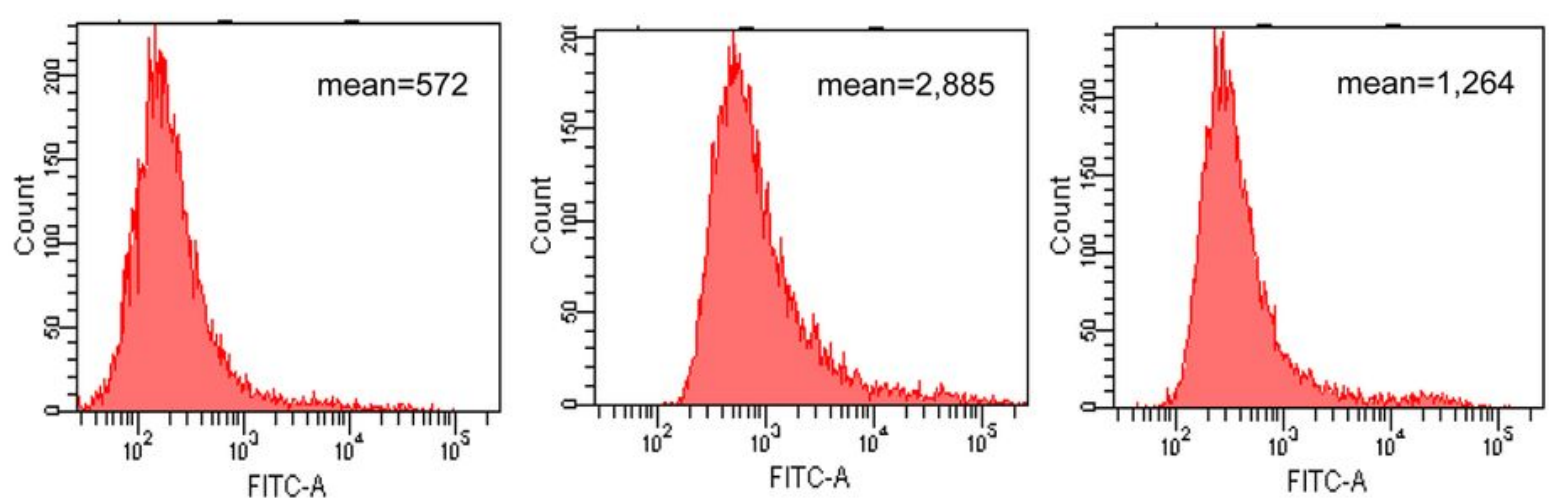

C
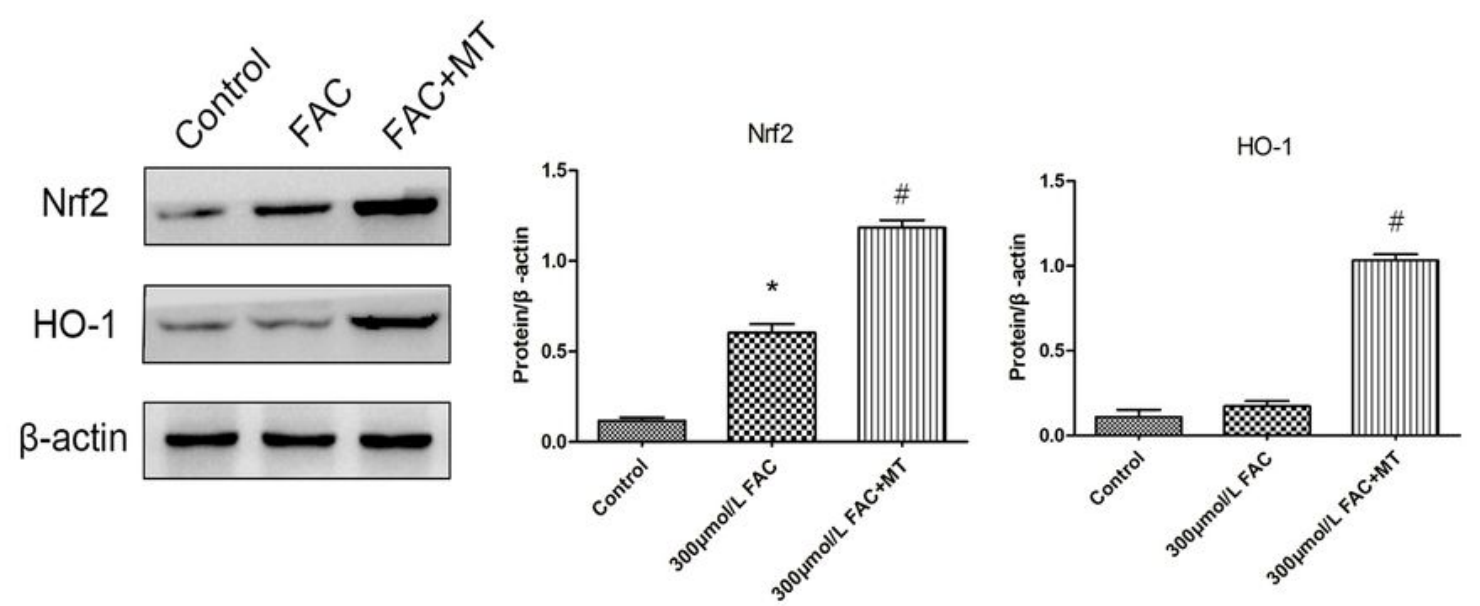

Figure 2

Melatonin protected hFOB1.19 cells against apoptosis via the Nrf2/HO-1 signaling pathway. A. Western blotting showing cleaved caspase 3 and BAX levels in hFOB1.19 cells treated with or without $100 \mu \mathrm{mol} / \mathrm{L}$ melatonin and incubated with $300 \mu \mathrm{mol} / \mathrm{L}$ FAC for $24 \mathrm{~h}$. The histogram shows the expression of caspase 
3 and BAX in the different groups. B. ROS generation was demonstrated by flow cytometry with dihydroethidium $(10 \mu \mathrm{M})$ after hFOB1.19 cells were treated for $24 \mathrm{~h}$. C. Nrf2 and HO-1 protein levels in hFOB1.19 cells demonstrated by western blotting. The histogram shows densitometric quantification of Nrf2 normalized to actin. Values represent the mean \pm S.E.M. of at least three independent experiments. $\star \mathrm{P}<0.05$ vs. control, \#P<0.05 vs. FAC treatment. 